\title{
Die Kennzeichnung von Spirituosen weinbaulichen Ursprungs in der EU - Eine Brücke zwischen Verbraucherschutz und Wirtschaftsinteressen
}

\author{
Werner Albrecht \\ Bundesministerium für Ernährung und Landwirtschaft, Referat 434 (Wein), 53123 Bonn, Germany
}

\begin{abstract}
Zusammenfassung. Das Spirituosen-Kennzeichnungsrecht in der EU stellt eine Brücke zwischen dem Verbraucherschutz und Wirtschaftsinteressen dar. Es ist ein eigenständiges Rechtsgebiet, das die Kennzeichnung auch von Spirituosen weinbaulichen Ursprungs regelt. Aus Sicht des Verbraucherschutzes weist es einige Unzulänglichkeiten auf und die ökonomischen Interessen der Hersteller haben Vorrang. So erlaubt das EU-Spirituosenrecht sog. zusammengesetzte Begriffe (Bsp. Brandy Likör, Gewürz Weinbrand) und sog. Anspielungen (Bsp. Apfellikör verfeinert mit Brandy), also die Verwendung von genau definierten und reservierten Spirituosennamen, obwohl es sich bei diesen Produkten nicht um die definierten Produktkategorien handelt. Spirituosen sind derzeit noch immer von der Angabe eines Zutatenverzeichnisses und der Nährwertdeklaration freigestellt. Diese Freistellung ist auf den Widerstand der Verbände der Alkoholwirtschaft (insbesondere der Wein- und Spirituosenwirtschaft) zurückzuführen, die beides ablehnen. Der gut informierte Verbraucher, der das besondere Geoschutz-System im Spirituosensektor kennt, kann allerdings Zusammensetzung und Kennzeichnungsvorschriften der Spirituosen mit einer geschützten geografischen Angabe (g.g.A.) in den sog. Technischen Unterlagen, die die Mitgliedstaaten veröffentlichen müssen, nachlesen. Eine Verbesserung der Informationen für die Verbraucher scheint in Zukunft möglich, nachdem insbesondere das Europäische Parlament jüngst die Brennwertangabe für alkoholische Getränke gefordert hat.
\end{abstract}

\begin{abstract}
The EU legislation concerning spirit drinks labelling constitutes a bridge between the interests of consumers to be protected against deception and being lead into error on one side and the economic interests of the spirit drinks producers and traders on the other side. This legislation containing also labelling provisions for spirit drinks of vitivinicultural origin is a particular legislation. With a view to consumer protection this legislation contains some deficits and gives priority to economic goals. For instance the EU spirit drinks legislation allows compound terms (e.g. Brandy Liqueur, spiced brandy) and allusions (e.g. apple liqueur refined by brandy), thus the use of defined and reserved spirit drinks terms, although these spirit drinks are not the defined product categories. Due to the economic interests being evaluated higher than consumer protection interests spirit drinks labelling does not include a list of ingredients or a nutrition declaration. The well-informed consumer has the possibility to get all the information about production process and ingredients of spirit drinks with a protected geographical indication (PGI) by consulting and reading the technical files which have to been published by the EU Member States. In future the information to consumers will be improved, especially after EU Parliament has adopted a motion or resolution asking Commission to introduce an energy declaration.
\end{abstract}

\section{Einführung}

In der Europäischen Union (EU) richtet sich die Kennzeichnung von Spirituosen weinbaulichen Ursprungs sowohl nach horizontalem Lebensmittelrecht - seit dem 13. Dezember 2014 ist dies die Lebensmittelinformationsverordnung (EU) Nr. 1169/2011, kurz LMIV genannt, - als auch nach produktspezifischem Spirituosenrecht. Seit dem 20. Mai 2008 ist dies die SpirituosenGrundverordnung (EG) Nr. 110/2008, davor, und zwar seit dem 15. Dezember 1989 galt die Vorgänger-Verordnung (EWG) Nr. 1576/89. Das EU-Spirituosenrecht enthält Begriffsbestimmungen für die folgenden Spirituosen weinbaulichen Ursprungs: Branntwein, Brandy oder Weinbrand, Tresterbrand oder Trester, Korinthenbrand oder Raisin Brandy, Traubenbrand und Weinhefebrand. Im Folgenden soll aufgezeigt werden, dass das SpirituosenKennzeichnungsrecht eine Brücke zwischen den
Interessen des Verbraucherschutzes und den wirtschaftlichen Interessen darstellt. Hersteller oder Anbieter von Spirituosen sind auf einen fairen Wettbewerb angewiesen. Das EU-Spirituosenrecht verfolgt auch Zielsetzungen der Gemeinsamen Agrarpolitik, z.B. die Sicherung des Absatzes von landwirtschaftlichen Ausgangserzeugnissen für Spirituosen, obwohl Spirituosen nach dem Vertrag über die Arbeitsweise der Europäischen Union (AEUVertrag) industrielle Erzeugnisse sind. Schließt schützt das EU-Spirituosenrecht auch geografische Angaben wie z.B. Cognac, Brandy de Jerez, Deutscher Weinbrand, Pfälzer Weinbrand oder Grappa.

\section{Horizontales Lebensmittelrecht}

Seit 13. Dezember 2014 gilt in der EU für alle Lebensmittel und damit auch für Spirituosen die Lebensmittelinformationsverordnung (EU) Nr. 1169/2011 (LMIV).

This is an Open Access article distributed under the terms of the Creative Commons Attribution License 4.0, which permits unrestricted use, distribution, and reproduction in any medium, provided the original work is properly cited. 
Für Spirituosen schreibt die LMIV folgende Kennzeichnungselemente vor (sog. verpflichtende Angaben): Bezeichnung, Allergien auslösende Zutaten, Menge bestimmter Zutaten (QUID), Nettofüllmenge, Name oder Firma und Anschrift des Lebensmittelunternehmers mit Sitz in der EU und vorhandener Alkoholgehalt in Volumenprozent. Im Spirituosensektor ist die Bezeichnung gemäß LMIV die nach VO (EG) Nr. 110/2008 vorgeschriebene Verkehrsbezeichnung. Für Spirituosen anders als für die meisten anderen Lebensmittel - sind nicht bzw. noch nicht vorgeschrieben: Zutatenverzeichnis, Mindesthalt-barkeitsdatum und Nährwertdeklaration. Die EU-Kommission ist bzw. war gehalten, bis 13. Dezember 2014 einen Bericht vorzulegen und darin der Frage nachzugehen, ob künftig auch alkoholische Getränke ein Zutatenverzeichnis und/oder die Nährwertdeklaration, ggf. nur die Brennwertangabe, aufweisen sollten. Dieser Bericht steht noch aus. Aus Verbraucherschutzsicht bewertet der Verfasser das Fehlen eines Zutatenverzeichnisses und auch der Brennwertangabe als Defizit (vgl. Abschnitt 3.2).

\section{Prinzipien des EU-Spirituosenrechtes}

Das EU-Spirituosenrecht definiert Produktkategorien (z.B. Branntwein oder Brandy) anhand von Spezifikationen und legt anhand dieser Spezifikationen nicht nur Mindestqualitätsstandards für diese Kategorien fest, sondern reserviert zugleich die hierfür verwendeten Namen als obligatorische Verkehrsbezeichnungen für diese Spirituosen. Werden alle Produktspezifikationen für die definierte Kategorie erfüllt, so muss die hierfür reservierte Bezeichnung als Verkehrsbezeichnung verwendet werden. Werden nicht alle Spezifikationen erfüllt, z.B. wird der Mindestalkoholgehalt nicht eingehalten, sondern unterschritten, muss die AuffangVerkehrsbezeichnung "Spirituose" verwendet werden. Nur für den Fall, dass eine Spirituose die Spezifikationen von mehr als zwei definierten Kategorien erfüllt, besteht eine Wahlmöglichkeit bei der Verkehrsbezeichnung oder die Möglichkeit, mehr als eine Verkehrsbezeichnung $\mathrm{zu}$ verwenden. Eine solche Wahlmöglichkeit könnte beispielsweise zwischen Branntwein und Weinbrand bestehen, wenn die zur Herstellung dieser Spirituose verwendeten Weindestillate alle zu weniger als $86 \%$ vol destilliert wurden, anschließend in Eichenholzfässern je nach Fassungsvermögen - mindestens sechs Monate oder ein Jahr gereift wurden und der Alkoholgehalt der trinkfertigen Spirituose mindestens 37,5\% vol beträgt. Die Festlegung von qualitativen Mindeststandards für die einzelnen Produktkategorien möchte zum einen den guten Ruf, den die in der EU hergestellten Spirituosen weltweit genießen sichern und zu einem fairen Wettbewerb unter den Spirituosenherstellern beitragen. Insider wissen, dass die Schaffung von spezifischem EU-Spirituosenrecht auch eine Antwort auf das EuGH-Urteil vom 20. Februar 1979 in der Rechtssache C-120/78 "Cassis de Dijon" war. Die Bundesmonopolverwaltung für Branntwein hatte einen aus Dijon stammenden Johannisbeerlikör mit einem Alkoholgehalt zwischen 15 und 20\% vol als nicht verkehrsfähig in Deutschland eingestuft, weil Liköre seinerzeit nach deutschem Recht einen Alkoholgehalt von mindestens $25 \%$ vol aufweisen mussten. Nach diesem EuGH-Urteil ist ein Produkt, das in einem EUMitgliedstaat verkehrsfähig ist, grundsätzlich in allen anderen EU-Mitgliedstaaten verkehrsfähig. Im Folgenden soll exemplarisch die Definition für Brandy näher erläutert und aus Verbraucherschutz- und ökonomischer Sicht bewertet werden.

\subsection{Begriffsbestimmung für "Brandy/Weinbrand"}

Das EU-Spirituosenrecht differenziert zwischen "Branntwein" (Anhang II Nr. 4 der Verordnung (EG) Nr. 110/2008) auf der einen und "Brandy" oder "Weinbrand" (Anhang II Nr. 5 a.a.O.) auf der anderen Seite. Gleichwohl basiert die Definition für Brandy auf der Begriffsbestimmung für Branntwein. Als zulässige Ausgangsstoffe für Brandy erlaubt das EU-Recht Branntwein, also Wein, Brennwein oder Rohbrand aus Wein, wobei alle drei Rohstoffe zu weniger als $86 \%$ vol destilliert werden müssen. Für die Herstellung von Brandy sind darüber hinaus - anders als für Branntwein - auch Weindestillate zulässig, die zu weniger als $94,8 \%$ vol destilliert wurden. Diese Weindestillate mit einer Alkoholstärke von bis zu 94,7\% vol dürfen allerdings nur höchstens $50 \%$ des Alkoholgehaltes des Fertigerzeugnisses ausmachen. Die Mindestreifezeit der Weindestillate in Eichenholzfässern beträgt ein Jahr oder sofern nur Eichenholzfässer mit einem Fassungsvermögen von weniger als 1.000 Litern verwendet werden, sechs Monate. Der Gehalt an flüchtigen Bestandteilen beträgt mindestens $125 \mathrm{~g} / \mathrm{hl}$ reinem Alkohol. Der Höchstgehalt an Methanol beträgt $200 \mathrm{~g} / \mathrm{hl}$ reinem Alkohol. Der Mindestalkoholgehalt von Brandy beträgt $36 \%$ vol. Der Zusatz von neutralem Ethylalkohol landwirtschaftlichen Ursprungs oder von rohstofffremdem Destillat landwirtschaftlichen Ursprungs ist auch in verdünnter Form nicht zulässig. Weiterhin ist der Zusatz von Aromen verboten. Eine Ausnahme sind lediglich sog. traditionelle Herstellungsverfahren, wobei es sich hier um den Zusatz von sog. Typagestoffen oder Bonifikateuren handelt, die in Deutschland nur aus bestimmten Ausgangsrohstoffen (z.B. Pflaumen, Walnüsse) und ausschließlich durch Mazeration mit Hilfe von Weindestillat mit einem Alkoholgehalt von weniger als $94,8 \%$ vol hergestellt sein müssen. Schließlich darf als einziger Farbstoff Zuckerkulör zur Anpassung der Farbe zugegeben werden.

\subsection{Bewertung der Begriffsbestimmung "Brandy"}

Im Ergebnis stellen 3 der 8 Qualitätskriterien für Brandy politische Kompromisse zwischen widerstreitenden ökonomischen Interessen der Brandy- bzw. Branntwein-Hersteller in den traditionellen Herstellerländern Frankreich, Italien, Spanien und Deutschland dar, die bei den Beratungen zur ersten EWG-Spirituosenverordnung in den 1980er Jahren zutage traten. Dabei wurden auch Verbraucherschutzinteressen einbezogen. Frankreich, Italien und Deutschland traten mit Nachdruck für eine Destillationsobergrenze von $86 \%$ vol ein, um sicherzustellen, dass die Weindestillate ausreichend sensorische Eigenschaften 
des Weines aufweisen. Demgegenüber plädierte Spanien entsprechend der einheimischen Tradition dafür, als Rohstoffgrundlage für Brandy eine Mischung von niedriggrädigen Weindestillaten mit höhergrädigeren Weindestillaten zuzulassen. Da Spanien von einer Reihe von anderen Mitgliedstaaten, u.a. von Griechenland, unterstützt wurde, kam letztlich der politische Kompromiss heraus, der die höhergrädigeren Weindestillate (weniger als 94,8\% vol) zulässt, aber mengenmäßig auf maximal $50 \%$ des Alkoholgehaltes des trinkfertigen Fertigerzeugnissen zulässt. Beim Mindestalkoholgehalt für den trinkfertigen Brandy wurde ebenfalls mit 36\% vol ein politischer Kompromisswert festgelegt. Spanien forderte einen Mindestalkoholgehalt von 34\% vol, Deutschland, Frankreich und Italien einen Mindestalkoholgehalt von 38\% vol. Die $36 \%$ vol liegen quasi in der Mitte. Schließlich wurde auch heftig über die Frage gestritten, ob im Falle eines Zusatzes von Neutralalkohol zu Brandy nicht nur die Bezeichnung "Brandy", sondern sogar die Produktion eines solchen Getränkes verboten werden sollte. Insbesondere in Deutschland gab und gibt es noch immer eine beträchtliche Produktion von "BranntweinVerschnitt". Dabei handelt es sich um eine Spirituose, deren Alkohol zu 10\% aus Weindestillat und zu 90\% aus neutralem Ethylalkohol besteht und die vor 1989 unter der Verkehrsbezeichnung "Branntwein-Verschnitt" und unter Phantasienamen wie "Goldbrand" oder "Goldkrone" vermarktet wurde. Seit dem 15. Dezember 1989 wird diese Spirituose EU-rechtskonform unter der AuffangVerkehrsbezeichnung "Spirituose" in den Verkehr gebracht. In Staaten des früheren Jugoslawiens war bzw. ist für eine vergleichbare Spirituose die Bezeichnung "domaci brandy" (inländischer Brandy) üblich. Politisch einigte man sich letztlich auf ein Bezeichnungsverbot, d.h. der definierte und reservierte Gattungsbegriff "Brandy" darf bei Verschnitten auf dem Etikett an keiner Stelle, auch nicht in Form eines zusammengesetzten Begriffes oder einer Anspielung (vgl. 5) erscheinen. Das Verbot, einem Brandy Aromen zuzusetzen, ist eine Qualitätsvorschrift, die einerseits einen fairen Wettbewerb unter den BrandyAnbietern sicherstellen und andererseits die Verbraucher vor Täuschung und Irreführung schützen soll. Die sensorischen Eigenschaften eines Brandys sollen mehr oder weniger ausschließlich aus der Fermentation der Ausgangserzeugnisse Wein oder Brennwein und der Destillation dieser Erzeugnisse herrühren. Der Zusatz von Aromen (Beispiel: Weinaromen) würde nicht nur Gärungs- und Destillationsfehler verdecken, sondern auch eine bessere Qualität vortäuschen. Als Ausnahme ist allerdings der Zusatz von sog. Bonifikateuren oder Typagestoffen zulässig. Dies ergibt sich aus Satz 2 in Anhang II Nr. 5 lit. d VO (EG) Nr. 110/2008: "Dies schließt traditionelle Herstellungsverfahren nicht aus". In Deutschland sind die zulässigen Bonifikateure in der (nationalen) Alkoholhaltige Getränke-Verordnung normiert. Zulässig sind Auszüge aus Eichenholzspänen, Walnüssen, Pflaumen oder Mandeln, die mit Weindestillat per Mazeration hergestellt und dem Brandy zur Geruchsund Geschmacksabrundung zugesetzt werden dürfen. In anderen EU-Mitgliedstaten sind weitere Bonifikateure zulässig, z.B. der Zusatz von Vanille-Auszügen. Aus Sicht des Verbraucherschutzes ist zu kritisieren, dass der Zusatz dieser Bonifikateure auf dem Etikett nicht angegeben werden muss. Das gleiche gilt auch für die Zugabe von Zucker oder anderen süßenden Erzeugnissen zur Abrundung des endgültigen Geschmacks von Brandy. Obwohl Artikel 5 Absatz 1 lit. e) VO (EG) Nr. 110/2008 der EU-Kommission aufgibt, den Höchstgehalt für Brände insgesamt oder auch differenziert nach den einzelnen Brand-Produktkategorien im Regelungsausschussverfahren mit Kontrolle festzulegen, hat die EU-Kommission bis dato nicht von dieser Ermächtigungsgrundlage Gebrauch gemacht. Letztlich hat die EU-Kommission das Vorhaben aufgegeben, weil sich keine qualifizierte Mehrheit der Mitgliedstaaten für bestimmte Höchstwerte abzeichnete. Mitgliedstaaten, in denen traditionell Brände oder Spirituosen, die keinen oder allenfalls geringste Mengen von Zucker oder anderen süßenden Erzeugnissen enthalten (z.B. 1 oder 2 g/Liter Fertigware), hergestellt und konsumiert werden, wie z.B. Whisky, Wodka oder Gin, hatten kein Verständnis für die Zugabe von süßenden Erzeugnissen zu Bränden bis $\mathrm{zu}$ oder sogar über $20 \mathrm{~g} /$ Liter Fertigware. Aus Sicht des Verbraucherschutzes muss auch bezüglich einer zulässigen Zuckerung zur Geschmacksabrundung kritisiert werden, dass ein solcher Zusatz nicht auf dem Etikett kenntlich gemacht werden muss. Überhaupt muss das Fehlen eines obligatorischen Zutatenverzeichnisses als Unzulänglichkeit im Hinblick auf einen ausreichenden Verbraucherschutz kritisiert werden. Spirituosen und alle anderen alkoholischen Getränke sollten nach der ersten Lebensmittel-Etikettierungsrichtlinie 79/112/EWG von der Angabe eines obligatorischen Zutatenverzeichnisses nur befristet bis 1982 freigestellt sein. Indes bestätigten die reformierte Lebensmittel-Etikettierungsrichtlinie 2000/13/ EG und die seit 13. Dezember 2014 geltende horizontale LMIV diese Freistellung vom Zutatenverzeichnis. Dass Spirituosen und andere alkoholischen Getränke - mit Ausnahme von Bier in Deutschland - in ihrer Etikettierung weder ein Zutatenverzeichnis noch eine Nährwertdeklaration tragen müssen, ist der guten Lobby-Arbeit der Verbände, welche die Interessen der Hersteller von Wein, Bier und Spirituosen vertreten, zurück zu führen. Sie führen immer wieder das Argument ins Feld, dass es für alkoholische Getränke detaillierte spezifische EUrechtliche Vorschriften gibt, denen der durchschnittlich informierte und verständige Verbraucher die Zusammensetzung entnehmen könne. Diese These ist allerdings nur bedingt richtig, weil - und darauf wird im nächsten Abschnitt 4 eingegangen - EU-Rechtsvorschriften auf anderen Gebieten (z.B. die Health Claims Verordnung) sogar eine angemessene Unterrichtung der Verbraucher verhindern. Die geltende LMIV (EU) Nr. 1169/2011 verpflichtet die Kommission zur Vorlage eines Berichtes, in dem diese prüfen und darlegen soll, ob auch für alkoholische Getränke künftig ein Zutatenverzeichnis oder eine Nährwertdeklaration vorgeschrieben werden soll. Die jüngste am 29. April 2015 vom Europäischen Parlament angenommene Entschließung zur Bekämpfung des Alkoholmissbrauchs lässt erwarten, dass die EUKommission zumindest bald einen Vorschlag für die Angabe des Brennwertes bei alkoholischen Getränken unterbreiten wird. 


\section{Health Claims Verordnung}

Die Verordnung (EG) Nr. 1924/2006 über gesundheitsund nährwertbezogene Angaben verbietet bei alkoholischen Getränken und damit auch bei Spirituosen derartige Angaben. So ist es z.B. einem BrandyHersteller, der seinem Erzeugnis keinen Zucker zur Abrundung des endgültigen Geschmacks zusetzt, nicht gestattet, Hinweise wie z.B. ohne Zucker oder zuckerfrei auf dem Etikett anzubringen. Denn diese Angaben sind nährwertbezogene Angaben, die bei alkoholischen Getränken ausnahmslos verboten sind. Der Hersteller eines Brandy, der keinen Zucker zur Geschmacksabrundung verwendet, hat zur Verbraucheraufklärung lediglich die Möglichkeit, entweder freiwillig ein Zutatenverzeichnis oder einen beschreibenden Hinweis wie z.B. "hergestellt ausschließlich aus Weindestillat und Wasser" auf das Etikett aufzubringen. Es darf jedoch aus Sicht des Autors bezweifelt werden, ob der durchschnittlich informierte und verständige Verbraucher diese Hinweise verstehen würde. Die EU-Kommission verfolgte mit der Health Claims Verordnung u.a. auch die Zielsetzung, den missbräuchlichen Alkoholkonsum einzudämmen bzw. zu verhindern. Traditionelle, seit vielen Jahren gebräuchliche Hinweise, wie z.B. "dieser Brandy erleichtert nach einer ausgiebigen Mahlzeit die Verdauung” oder "dieser Brandy ist wohltuend oder bekömmlich", sind nicht mehr zulässig. Bezüglich "bekömmlich" gibt es ein EuGHUrteil (Urteil vom 6. September 2012; RS C-544/10), das die Unzulässigkeit dieses Begriffs bei Wein ausdrücklich bestätigt.

\section{Zusammengesetzte Begriffe und Anspielungen}

Nach Artikel 9 Absätze 1 bis 4 der SpirituosenGrundverordnung (EG) Nr. 110/2008 dürfen die für bestimmte Produktkategorien oder Spirituosen mit einer geografischen Angabe reservierten Bezeichnungen nur verwendet werden, wenn die fragliche Spirituose sämtliche in der Begriffsbestimmung niedergelegten Produktspezifikationen einhält. Art. 9 Abs. 4 a.a.O verweist jedoch auf die Ausnahme des Art. 10 Abs. 1 a.a.O, nämlich auf die sog. zusammengesetzten Begriffe und Anspielungen. Nach mehrjährigem Streit im EU-Spirituosenausschuss in Brüssel, ob z.B. Whisky Likör oder Brandy Likör bzw. Bezeichnungen wie Apfel Brandy oder Gewürz Tresterbrand zulässig sind, hat die EU-Kommission die Durchführungsverordnung (EU) Nr. 716/2013 erlassen, die Definitionen und Verwendungsvoraussetzungen für die zusammen-gesetzten Begriffe und Anspielungen enthält.

\subsection{Zusammengesetzte Begriffe}

Nach Art. 2 lit. c DVO (EU) Nr. 716/2013 versteht man unter einem "zusammengesetzten Begriff" die Kombination aus einem geschützten Spirituosennamen (entweder eine der 47 Gattungsbezeichnungen oder eine der über 300 geografischen Angaben, die im Anhang III der VO (EG) Nr. 110/2008 aufgeführt sind) mit dem Namen eines oder mehrerer anderer Lebensmittel und/oder dem Begriff "Likör". Bei den Lebensmittelnamen handelt es sich um andere als Spirituosennamen und andere Namen als die Rohstoffe, die für die jeweilige Produktkategorie gemäß Definition verwendet werden. So wären Bezeichnungskombinationen wie z.B. Traubenlikör oder Getreide Wodka keine zusammengesetzten Begriffe im Sinne des EU-Spirituosenrechtes, weil im ersten Falle Trauben, Traubensaft oder Traubenaroma zur Aromatisierung des Likörs verwendet werden und im zweiten Falle der zur Herstellung des Wodka verwendete Ethylalkohol landwirtschaftlichen Ursprungs aus Getreide stammt. Im Übrigen handelt es sich bei den beiden genannten Beispielen um einen echten Likör bzw. um einen echten Wodka im Sinne der einschlägigen Begriffsbestimmungen. Demgegenüber wären "Apfel Brandy", "Apfel Sahne Brandy", "Brandy Likör" oder "Apfel Brandy Likör" Beispiele für sog. zusammengesetzte Begriffe. Art. 3 DVO (EU) Nr. 716/2013 legt die Verwendungsvoraussetzungen fest. Unter anderem ist ein zusammengesetzter Begriff keine Verkehrsbezeichnung, so dass ein Erzeugnis mit dem zusammengesetzten Begriff "Brandy Likör" zusätzlich mit der Verkehrsbezeichnung "Likör" vermarktet werden muss. Im Ergebnis wird der Begriff "Likör" in der Etikettierung zwei Mal verwendet, wobei der zusammengesetzte Begriff "Brandy Likör" auf dem Frontetikett und die Verkehrsbezeichnung "Likör" auf dem Rücketikett angebracht werden darf. Weiterhin gibt es genaue Formvorschriften wie gleiches Schriftbild, gleiche Schriftgröße und gleiche Farbe sowie das Gebot, die einzelnen Namen im zusammengesetzten Begriff nicht durch textliche oder bildliche Elemente zu unterbrechen. Vorgeschrieben ist auch, dass die Schriftgröße des zusammengesetzten Begriffs nicht größer sein darf als die der Verkehrsbezeichnung. Dazu ein Beispiel: In den USA werden zunehmend sog. flavoured Spirituosenkategorien hergestellt wie z.B. Honig Whiskeys (honey whiskey) oder Gewürzbrandy (spiced brandy). Diese zusammengesetzten Begriffe werden marketingmäßig plakativ und in großen Buchstaben auf das Frontetikett gesetzt. Die EU-rechtliche Vorschrift "keine größere Schriftgröße als die Verkehrsbezeichnung" bedeutet, dass die Verkehrsbezeichnung "Spirituose" - in der Regel handelt es sich nicht um Liköre, sondern um nicht zu stark gesüßte und aromatisierte Spirituosen - mindestens so groß wie der zusammengesetzte Begriff "spiced brandy" sein muss. Bei den EU-Vorschriften zu den sog. zusammengesetzten Begriffen handelt es sich um höchst komplizierte Vorschriften, die nicht so ohne weiteres von den Spirituosenherstellern verstanden und erst recht aus Sicht des Verfassers keinen Mehrwert für den Verbraucherschutz bringen. Die Kompliziertheit rührt auch daher, dass in der englischen Sprache eine Aneinanderreihung von Substantiven (Nomen) leicht verständlich ist, in den romanischen Sprachen hingegen die einzelnen Elemente mit Präpositionen oder Füllwörtern kombiniert werden müssen, um verständlich zu sein (Beispiele auf französisch: liqueur au brandy oder liqueur à base du brandy). Diese Möglichkeit der Ergänzung um Präpositionen wird durch folgende Ausnahmeregelung von der Vorschrift, dass die Elemente eines zusammengesetzten Begriffs nicht durch textliche oder bildliche Elemente unterbrochen werden dürfen, gewährleistet: “.., der bzw. 
die nicht Teil des zusammengesetzten Begriffs ist, ..." (Einschub in Art. 3 Abs. 4 Satz 2 DVO (EU) Nr. 716/2013).

Im Unterschied zu Anspielungen (vgl. 5.2) gelten die Regeln für zusammengesetzte Begriffe nur bei einer Verwendung in der Etikettierung von alkoholischen Getränken (Spirituosen, Wein, Bier, Fruchtwein etc.), nicht jedoch für alle anderen Lebensmitteln wie z.B. Kuchen, Eis oder Joghurt. Abschließend ist darauf hinzuweisen, dass zusammengesetzte Begriffe, die in der SpirituosenGrundverordnung (EG) Nr. 110/2008 genannt oder im Anhang II definiert werden, keine zusammengesetzten Begriffe im Sinne der DVO (EU) Nr. 716/2013 sind. Dies erklärt sich bereits aus dem Umstand, dass Vorschrift der Basisverordnung höherwertiges Recht und zugleich Spezialrecht gegenüber dem Recht der DVO (EU) Nr. 716/2013 darstellen. Die wichtigsten Beispiele sind die in Anhang II Nr. 32 lit. d) der VO (EG) Nr. 110/2008 expressis verbis genannten Produkte "prune-brandy", "orange-brandy", "apricoat-brandy" oder "cherry-brandy", bei denen es sich um Fruchtaromaliköre handelt, die auf Basis von neutralem Ethylalkohol landwirtschaftlichen Ursprungs hergestellt werden.

\subsection{Anspielungen}

Gemäß Art. 2 lit. d) DVO (EU) Nr. 716/2013 versteht man unter einer "Anspielung" die direkte oder indirekte Bezugnahme auf eine oder mehrere Spirituosenkategorien, die nicht Teil eines zusammengesetzten Begriffs oder in einer Zutatenliste stehen. Die wichtigste Verwendungsvoraussetzung gemäß Art. 4 a.a.O. ist das Gebot, die Anspielung in einer getrennten Zeile wie die Verkehrsbezeichnung zu schreiben. Ein Beispiel hierfür wäre "Eierlikör" (hergestellt auf Basis von neutralem Ethylalkohol landwirtschaftlichen Ursprungs) und abgesetzt in einer zweiten Zeile "verfeinert mit Brandy".

\subsection{Vergleich zusammengesetzter Begriff (ZB) versus Anspielung(en) (A)}

Die folgende Tabelle zeigt die wesentlichen Unterschiede zwischen einem ZB und einer A auf.

\section{Künftige Entwicklungen}

Die geltende Spirituosen-Grundverordnung (EG) Nr. 110/2008 soll in Kürze an den Vertrag von Lissabon angepasst werden. Insbesondere geht es hier um die Frage, welche Durchführungsvorschriften die EU-Kommission künftig im Wege einer sog. delegierten Verordnung - hier haben der Rat und das EP innerhalb einer Frist von 2 bzw. 4 Monaten Kontrollrechte einschließlich eines Vetorechts und welche in Form einer sog. Durchführungsverordnung - hier geht es um den Vollzug von EU-Recht in den Mitgliedstaaten und die Mitgliedstaaten haben die Möglichkeit, im Rat die Durchführungsverordnung zu verhindern - erlassen werden sollen. Diese Anpassung an den Lissabon-Vertrag (Vertrag über die Arbeitsweise der Europäischen Union) bietet eine gute Gelegenheit, auch inhaltliche Korrekturen an der geltenden SpirituosenGrundverordnung vorzunehmen. So könnte spezielles Kennzeichnungsrecht geschaffen werden, das Vorrang vor
Tabelle 1. Vergleich ZB vs. A.

\begin{tabular}{|c|c|}
\hline $\mathbf{Z B}$ & $\mathbf{A}$ \\
\hline $\begin{array}{l}\text { Vorschriften gelten nur bei } \\
\text { Verwendung in der } \\
\text { Etikettierung von } \\
\text { alkoholischen Getränken }\end{array}$ & $\begin{array}{l}\text { Vorschriften gelten bei } \\
\text { Verwendung von } \\
\text { Spirituosennamen in der } \\
\text { Etikettierung von allen } \\
\text { Lebensmitteln (Getränke, } \\
\text { Kuchen, Gebäck, Süßwaren) }\end{array}$ \\
\hline $\begin{array}{l}\text { ein einziger } \\
\text { Spirituosen-name } \\
\text { (Gattungsbegriff } \\
\text { oder geografische } \\
\text { Angabe); Ausnahme: } \\
\text { Spirituosenname + Likör }\end{array}$ & $\begin{array}{l}\text { ein oder mehrere } \\
\text { Spirituosennamen }\end{array}$ \\
\hline in einer Zeile & $\begin{array}{l}\text { in getrennter Zeile zur } \\
\text { Verkehrsbezeichnung }\end{array}$ \\
\hline $\begin{array}{l}\text { einheitliche Schrift (Typ, } \\
\text { Größe und Farbe) }\end{array}$ & - \\
\hline $\begin{array}{l}\text { ZB darf nicht größer als } \\
\text { Verkehrsbezeichnung sein }\end{array}$ & $\begin{array}{l}\text { Anspielung muss kleinere } \\
\text { Schriftgröße als } \\
\text { Verkehrsbezeichnung haben }\end{array}$ \\
\hline $\begin{array}{l}\text { ZB ist keine } \\
\text { Verkehrsbezeichnung, } \\
\text { daher wird in der Regel } \\
\text { "Spirituose" oder "Likör" } \\
\text { - je nach Zuckergehalt - } \\
\text { als Verkehrsbezeichnung } \\
\text { ergänzt. }\end{array}$ & $\begin{array}{l}\text { Anspielung kann eine } \\
\text { Verkehrsbezeichnung } \\
\text { ergänzen, d.h. keine } \\
\text { Doppelung des Namens } \\
\text { "Likör" wie bei einer ZB }\end{array}$ \\
\hline
\end{tabular}

anderen horizontalen lebensmittelrechtlichen Vorschriften wie z.B. die LMIV oder die Health Claims Verordnung (HCV) hat. Um die Verbraucher über die Bedeutung von Altersprädikaten gerade bei Brandy wie z.B. VSOP, $\mathrm{XO}$ etc. aufzuklären, sollte die EU-Kommission ggf. eine delegierte Verordnung erlassen.

Die im EU-Spirituosenrecht für Spirituosen weinbaulichen Ursprungs festgelegten Begriffsbestimmungen (u.a. für Branntwein, Brandy, Trester, Traubenbrand und Weinhefebrand) haben sich bewährt und stellen in qualitativer Hinsicht einen guten Kompromiss dar. Vorschläge, z.B. den Mindestalkoholgehalt für Brandy abzusenken, sollten nach Meinung des Verfassers nicht akzeptiert werden.

Das Europäische Parlament hat am 29. April 2015 in einer Entschließung zur Bekämpfung des Alkoholmissbrauchs bzw. für eine neue EU-Alkoholstrategie die Kommission auch aufgefordert, baldmöglichst die Brennwertangabe für alkoholische Getränke vorzuschreiben. Der Verfasser ist der Auffassung, dass die Brennwertangabe durchaus den Verbraucher besser über die Inhaltsstoffe von alkoholischen Getränken informieren kann. So könnte eine nach Alkohol und Zucker getrennte Brennwertangabe bei Bränden wie z.B. Brandy den Verbraucher darüber informieren, ob ein Brandy zur Geschmacksabrundung gesüßt wurde oder nicht.

\section{Schutz geografischer Angaben}

Das EU-Spirituosenrecht enthält einen eigenständigen Schutz von geografischen Angaben. Im Unterschied zum Wein- oder sonstigen Lebensmittelsektor differenziert das 


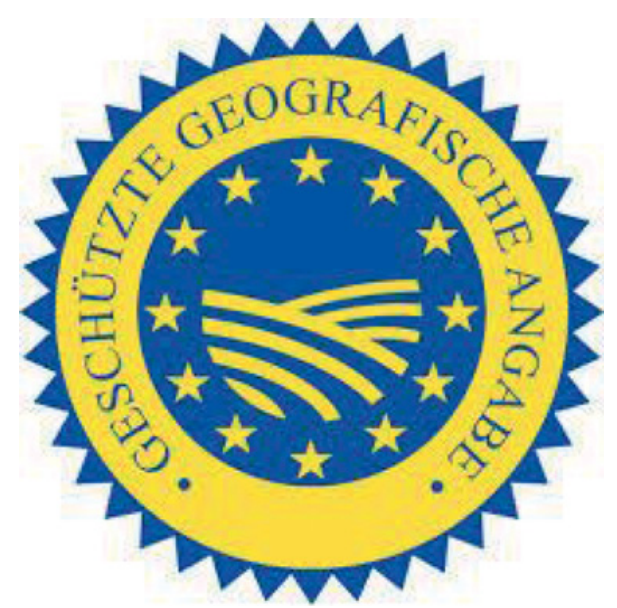

Abb 1. EU-Symbol geschützte geografische Angabe.

EU-Spirituosenrecht nicht zwischen einer "geschützten geografischen Angabe (g.g.A.)" und einer "geschützten Ursprungsbezeichnung (g.U.)". Weiterhin differenziert das EU-Spirituosenrecht zwischen den sog. etablierten geografischen Angaben, die am 20. Februar 2008, also zum Zeitpunkt des formellen Inkrafttretens der reformierten Spirituosen-Verordnung (EG) Nr. 110/2008 bereits im Anhang III a.a.O. eingetragen waren und den sog. neuen geografischen Angaben, die später, also nach dem 20. Februar 2008 in den Anhang III a.a.O. eingetragen wurden. Beispiele für etablierte geografische Angaben sind "Cognac", "Brandy de Jerez", "Deutscher Weinbrand" oder "Pfälzer Weinbrand". Ein Beispiel für eine neue geografische Angabe ist "Pisco" (aus Peru) hierbei handelt es sich um einen Traubenbrand -, wobei Pisco aus Chile per bilateralem Abkommen der EU mit Chile ebenfalls als geografische Angabe in der EU geschützt ist. Die Mitgliedstaaten waren verpflichtet, für die etablierten geografischen Angaben bis zum 20. Februar 2015 sog. technische Unterlagen mit den Produktspezifikationen vorzulegen. Soweit bekannt, hat Frankreich keine technische Unterlage für "französischen Brandy" eingereicht, Deutschland hat fristgerecht für "Deutscher Weinbrand" und für "Pfälzer Weinbrand" eine technische Unterlage eingereicht. Diese technischen Unterlagen werden derzeit von der EU-Kommission geprüft. Würde man die Kriterien der in der EU für sonstige Lebensmittel geltenden Verordnung (EU) Nr. 1151/2012 heranführen, wäre "Deutscher Weinbrand" eine g.g.A. sowie "Pfälzer Weinbrand" und "Cognac" eine g.U.

Im Unterschied zum sonstigen Lebensmittelsektor muss im Spirituosensektor das EU-Logo einer g.g.A. nicht obligatorisch verwendet werden. Der Verfasser ist jedoch der Auffassung, dass die Hersteller von Spirituosen mit einer g.A. das EU-Logo verwenden sollten, um es in der EU bekannter zu machen.

Im Unterschied zu Gattungsbegriffen kann der Verbraucher bei Spirituosen mit einer g.g.A. die Inhaltstoffe und die Zusammensetzung und das Herstellungsverfahren in den veröffentlichten technischen Unterlagen nachlesen.
In Deutschland sind die technischen Unterlagen auf der Webseite der Bundesanstalt für Landwirtschaft und Ernährung - http://www.ble.de / Kontrolle / EUQualitätskennzeichen / geschützte geografische Angaben für Spirituosen - veröffentlicht.

Die EU-Kommission prüft derzeit im Rahmen der vom neuen Agrarkommissar Phil Hogan bzw. vom Präsidenten der EU-Kommission Jean-Claude Juncker angestoßenen Vereinfachung der Gemeinsamen Agrarpolitik, ob der Schutz der geografischen Angaben im Spirituosensektor künftig in einer vereinheitlichen einzigen GeoschutzVerordnung gewährleistet werden soll. Der Verfasser ist der Auffassung, dass es für den Rechtsunterworfenen besser wäre, den Geoschutz weiterhin in der Spirituosenverordnung und damit im Produktrecht $\mathrm{zu}$ belassen. Es gibt auch eine Reihe von Besonderheiten des Spirituosensektors, welche die getrennte Regelung weiterhin rechtfertigen.

\section{Zusammenfassung}

Das Zusammenwirken von horizontalem EULebensmittelrecht und dem produktspezifischen Spirituosenrecht hat sich grundsätzlich bewährt. Für Spirituosen mit Ursprung in der EU gelten hohe Qualitätsstandards, die dazu geführt haben, dass EU-Spirituosen auf dem Weltmarkt einen exzellenten Ruf genießen. Diesen Ruf gilt es zu verteidigen. Gleichwohl bestehen noch Defizite in Bezug auf den Verbraucherschutz bzw. für eine noch bessere Information der Verbraucher über Inhaltsstoffe, die der durchschnittlich informierte und verständige Verbraucher nicht vermuten würde. Dazu zählen z.B. Bonifikateure bei Weinbrand oder Zucker zur Geschmacksabrundung. Werden Bonifikateure verwendet, die aus allergenen Zutaten wie z.B. Walnüssen oder Mandeln hergestellt werden, so sind diese schon heute auch außerhalb eines Zutatenverzeichnisses in der Etikettierung kenntlich zu machen. Der Verfasser ist optimistisch, dass hier auf EU-Ebene mittelfristig weitere Verbesserungen im Sinne des Verbraucherschutzes erfolgen.

\section{Literaturhinweise}

Der Autor hat in der Vergangenheit schon wiederholt Fachaufsätze zum EU-Spirituosenrecht verfasst oder Vorträge vor Brennerei- oder Spirituosenverbänden gehalten. Alle Fachaufsätze hier aufzuführen, würde den Rahmen dieser Abhandlung sprengen. Betont werden soll, dass die in der vorliegenden Abhandlung geäußerten Auffassungen persönliche Auffassungen des Autors sind und keine offiziellen Positionen des Bundesministeriums für Ernährung und Landwirtschaft oder gar der Bundesregierung darstellen.

Die folgende Literatur- bzw. Quellenübersicht beschränkt sich auf wenige EU-Rechtsakte. Die zitierten EuGH-Urteile können über Internet-Suchmaschinen durch Eingabe des jeweiligen Stichwortes (z.B. Cassis de Dijon) leicht gefunden werden. 
[1] Lebensmittelinformationsverordnung (LMIV) (EU) Nr. 1169/, konsolidierte Fassung unter http: //eurlex.europa. eu/legal-content/DE/TXT/PDF/? uri $=$ CELEX:02011R1169-20140219\&rid=1)

[2] Spirituosen-Grundverordnung (EG) Nr. 110/2008, konsolidierte Fassung unter http://eur-lex. europa.eu/legal-content/DE/TXT/PDF/?uri= CELEX : 02008R0110-20150214\&rid=1

[3] Spirituosen-Durchführungsverordnung (EU) Nr. 716/2013, konsolidierte Fassung unter http:// eur-lex. europa.eu/legal-content/DE/TXT/ $\mathrm{PDF} /$ ?uri=CELEX : 02013R0716-20150101\&rid=1
[4] Health Claims Verordnung (HCVO) (EG) Nr. 1924/2006, konsolidierte Fassung unter http:// eur-lex. europa.eu/legal-content/DE/TXT/ $\mathrm{PDF} /$ ?uri=CELEX : 02006R1924-20141213\&rid=1

[5] Verordnung (EU) Nr. 1151/2012 über Qualitätsregelungen für Agrarprodukte und Lebensmittel, konsolidierte Fassung unter http:// eur-lex.europa.eu/legal-content/EN/TXT/ $\mathrm{PDF} /$ ?uri=CELEX : 02012R1151-20130103\&qid= $1431100661661 \&$ from $=\mathrm{DE}$ 